\title{
POSITIVITY OF THE TRANSMUTATION OPERATORS AND ABSOLUTE CONTINUITY OF THEIR REPRESENTING MEASURES FOR
}

\section{A ROOT SYSTEM ON $\mathbb{R}^{d}$}

\author{
Khalifa Trimèche \\ University of Tunis El Manar \\ Faculty of Sciences of Tunis \\ Department of Mathematics, Campus \\ 2092, Tunis, TUNISIA
}

\begin{abstract}
We consider the transmutation operators $V_{k}, V_{k}^{W}$ and ${ }^{t} V_{k}{ }^{t} V_{k}^{W}$ associated respectively with the Cherednik operators and the Heckman-Opdam theory, called also in [9], [10] the trigonometric Dunkl intertwining operators and their dual paper. In this paper we prove that the operators $V_{k}, V_{k}^{W}$ and ${ }^{t} V_{k},{ }^{t} V_{k}^{W}$ are positivity preserving and allows positive integral representations. Next we study the absolute continuity of their representing measures and we deduce that the Opdam-Cherednik kernel and the Heckman-Opdam hypergeometric function are positive definite.
\end{abstract}

AMS Subject Classification: 33E30,33C67, 47B34, 51F15

Key Words: Cherednik operators, Heckman-Opdam theory, root system on $\mathbb{R}^{d}$, transmutation operators, trigonometric Dunkl intertwining operators, absolute continuity of the representing measures

\section{Introduction}

We consider the differential-difference operator $T_{j}, j=1,2, \ldots, d$ on $\mathbb{R}^{d}$ associated with a root system $\mathcal{R}$, a Weyl group $W$ and a multiplicity function $k$, 
introduced by I. Cherednik [2], and called the Cherednik operators in the literature. These operators play a crucial role in the theory of Heckman-Opdam's hypergeometric functions; which generalizes the theory of Harish-Chandra's spherical functions on Riemann symmetric spaces (see [4],[5],[7]).

To study in [10],[12] the harmonic analysis associated with the Cherednik operator and the Heckman-Opdam theory, the author has introduced in [9] the transmutation operators $V_{k}, V_{k}^{W}$ called also the trigonometric Dunkl intertwining operators and their dual ${ }^{t} V_{k},{ }^{t} V_{k}^{W}$. In many situations to solve problems in this harmonic analysis we need the positivity of these operators, and the absolute continuity of their representing measures. This property is not yet proved in the general case, it is obtained only in the one dimensional case (see [3],[11]).

In this paper we prove first that for all $g$ in $\mathcal{E}\left(\mathbb{R}^{d}\right)$ (the space of $C^{\infty}$-functions on $\mathbb{R}^{d}$ ) we have

$$
\forall x \in \mathbb{R}^{d}, V_{k}(g)(x)=\int_{\mathbb{R}^{d}} g(y) d \mu_{x}(y),
$$

where $\mu_{x}$ is a positive measure with compact support contained in the closed ball $B(0,\|x\|)$ of center 0 and radius $\|x\|$, and of norm equal to 1 .

And for all function $f$ in $\mathcal{D}\left(\mathbb{R}^{d}\right)$ (the space of $C^{\infty}$-functions on $\mathbb{R}^{d}$, with compact support) we have

$$
\forall y \in \mathbb{R}^{d},{ }^{t} V_{k}(f)(y)=\int_{\mathbb{R}^{d}} f(x) d \nu_{y}(x),
$$

where $\nu_{y}$ is a positive measure with support in the set $\left\{x \in \mathbb{R}^{d} ;\|x\| \geq\|y\|\right\}$.

From the previous results we have deduced that for all functions $g$ in $\mathcal{E}\left(\mathbb{R}^{d}\right)^{W}$ (the subspace of $\mathcal{E}\left(\mathbb{R}^{d}\right)$ of $W$-invariant functions) and $f$ in $\mathcal{D}\left(\mathbb{R}^{d}\right)^{W}$ (the subspace of $\mathcal{D}\left(\mathbb{R}^{d}\right)$ of $W$-invariant functions) we have

$$
\forall x_{i} n \mathbb{R}^{d}, V_{k}^{W}(g)(x)=\int_{\mathbb{R}^{d}} g(y) d \mu_{x}^{W}(y),
$$

and

$$
\forall y \in \mathbb{R}^{d},{ }^{t} V_{k}^{W}(f)(y)=\int_{\mathbb{R}^{d}} f(x) d \nu_{y}^{W}(y),
$$

where

$$
\mu_{x}^{W}=\frac{1}{|W|} \sum_{w \in W} \mu_{w x}
$$

and

$$
\nu_{y}^{W}=\frac{1}{|W|} \sum_{w \in W} \nu_{w y} .
$$


Next, we establish that for all $x \in \mathbb{R}_{\text {reg }}^{d}$ (the regular part of $\mathbb{R}^{d}$ ) and $y \in \mathbb{R}^{d}$, the measures $\mu_{x}, \mu_{x}^{W}$ and $\nu_{y}, \nu_{y}^{W}$ are absolute continuous with respect to the Lebesgue measure on $\mathbb{R}^{d}$. More precisely there exist positive functions $\mathcal{K}(x, y)$ and $\mathcal{K}^{W}(x, y)$ such that

$$
\begin{gathered}
d \mu_{x}(y)=\mathcal{K}(x, y) d y, \\
d \mu_{x}^{W}(y)=\mathcal{K}^{W}(x, y) d y, \\
d \nu_{y}(y)=\mathcal{K}(x, y) \mathcal{A}_{k}(x) d x, \\
d \nu_{y}^{W}(x)=\mathcal{K}(x, y) \mathcal{A}_{k}(x) d x,
\end{gathered}
$$

where $\mathcal{A}_{k}(x)$ is a weight function on $\mathbb{R}^{d}$ which will be given in the following section (see (2.6)).

The functions $y \rightarrow \mathcal{K}(x, y)$ and $y \rightarrow \mathcal{K}^{W}(x, y)$ have their support contained in the closed ball $B(0,\|x\|)$, and satisfy

$$
\int_{\mathbb{R}^{d}} \mathcal{K}(x, y) d y=1
$$

and

$$
\int_{\mathbb{R}^{d}} \mathcal{K}^{W}(x, y) d y=1
$$

As applications of the previous results, we prove that for all $\lambda \in \mathbb{C}^{d}$, the Opdam-Cherednik kernel $G_{\lambda}$ and the Heckman-Opdam hypergeometric function $F_{\lambda}$ possess the following integral representations

$$
\forall x \in \mathbb{R}_{r e g}^{d}, G_{\lambda}(x)=\int_{\mathbb{R}^{d}} \mathcal{K}(x, y) e^{-i\langle\lambda, y\rangle} d y,
$$

and

$$
\forall x \in \mathbb{R}_{r e g}^{d}, F_{\lambda}(x)=\int_{\mathbb{R}^{d}} \mathcal{K}^{W}(x, y) e^{-i\langle\lambda, y\rangle} d y,
$$

The relations (1.13), (1.14) show that the functions $G_{\lambda}$ and $F_{\lambda}$ are positive definite.

\section{The Cherednik Operators on $\mathbb{R}^{d}$ and Their Eigenfunctions}

\subsection{The Root System and the Multiplicity Function}

We consider $\mathbb{R}^{d}$ with the standard basis $\left\{e_{1}, e_{2}, \ldots, e_{d}\right\}$, and the inner product $\langle.,$.$\rangle for which this basis is orthonormal.$ 
We take the root system $R \subset \mathbb{R}^{d} \backslash\{0\}$ given by

$$
\mathcal{R}=\left\{ \pm \alpha_{i}, \pm 2 \alpha_{i} ; i=1,2, \ldots, d\right\} .
$$

We denote by $\mathcal{R}_{+}$the set of positive roots

$$
\mathcal{R}_{+}=\left\{\alpha_{i}, 2 \alpha_{i}, i=1,2, \ldots, d\right\},
$$

and by $\mathcal{R}_{+}^{0}$ the set of positive indivisible roots i.e. the roots $\alpha \in \mathcal{R}_{+}$such that $\frac{\alpha}{2} \notin \mathcal{R}_{+}$. Then we have

$$
\mathcal{R}_{+}^{0}=\left\{\alpha_{i} ; i=1,2, \ldots, d\right\} .
$$

For $\alpha \in \mathcal{R}$, we consider

$$
r_{\alpha}(x)=x-\langle\breve{\alpha}, x\rangle \alpha, \text { with } \breve{\alpha}=\frac{2}{\|\alpha\|^{2}} \alpha,
$$

the reflection in the hyperplane $H_{\alpha} \subset \mathbb{R}^{d}$ orthogonal to $\alpha$. The reflections $r_{\alpha}, \alpha \in \mathcal{R}$ generate a finite group $W \subset O(d)$ called the Weyl group associated with $\mathcal{R}$.

The multiplicity function associated with $\mathcal{R}$ is a function $k: \mathcal{R} \rightarrow[0,+\infty[$ which is invariant under the action of the group $W$.

Let $\mathfrak{a}_{+}$be the positive Weyl chamber given by

$$
\mathfrak{a}_{+}=\left\{x \in \mathbb{R}^{d} ; \forall \alpha \in \mathcal{R}_{+},\langle\alpha, x\rangle>0\right\},
$$

we denote by $\overline{\mathfrak{a}}_{+}$its closure. Let also $\mathbb{R}_{r e g}^{d}$ be the subset of regular elements in $\mathbb{R}^{d}$, i.e. those elements which belong to no hyperplanes $H_{\alpha_{i}}=\left\{x \in \mathbb{R}^{d} ;\left\langle\alpha_{i}, x\right\rangle=\right.$ $0\}, i=1,2, \ldots, d$.

Let $\mathcal{A}_{k}$ denotes the weight function

$$
\forall x \in \mathbb{R}^{d}, \mathcal{A}_{k}(x)=\prod_{\alpha \in \mathcal{R}_{+}}\left|\sinh \left\langle\frac{\alpha}{2}, x\right\rangle\right|^{2 k(\alpha)} .
$$

This function can also be written in the form

$$
\begin{aligned}
\forall x \in \mathbb{R}^{d} ; \mathcal{A}_{k}(x)=\left(\prod_{i=1}^{d} 2^{2 k\left(2 \alpha_{i}\right)}\right) & \left(\prod_{i=1}^{d}\left|\sinh \left\langle\frac{\alpha_{i}}{2}, x\right\rangle\right|^{2\left(k\left(\alpha_{i}\right)+k\left(2 \alpha_{i}\right)\right.}\right) \\
& \times\left.\cosh \left\langle\frac{\alpha_{i}}{2}, x\right\rangle\right|^{2 k\left(2 \alpha_{i}\right)} .
\end{aligned}
$$

In this paper we suppose that the following conditions are satisfied:

1. The vectors $\alpha_{1}, \alpha_{2}, \ldots, \alpha_{d}$ are linearly independent.

2. We have

$$
\forall \alpha \in \mathcal{R}_{+}, k(\alpha)+k(2 \alpha)>0 .
$$




\subsection{The Cherednik Operators}

The Cherednik operators $T_{j}, j=1,2, \ldots, d$, on $\mathbb{R}^{d}$ associated with the Weyl group $W$ and the multiplicity function $k$ are defined for $f$ of class $C^{1}$ on $\mathbb{R}^{d}$ and $x \in \mathbb{R}_{r e g}=\mathbb{R}^{d} \backslash \bigcup_{i=1}^{d} H_{\alpha_{i}}$ by

$$
T_{j} f(x)=\frac{\partial}{\partial x_{j}} f(x)+\sum_{\alpha \in \mathcal{R}_{+}} \frac{k(\alpha) \alpha^{j}}{1-e^{-\langle\alpha, x\rangle}}\left\{f(x)-f\left(r_{\alpha} x\right)\right\}-\rho_{j} f(x),
$$

with

$$
\rho_{j}=\frac{1}{2} \sum_{\alpha \in \mathcal{R}_{+}} k(\alpha) \alpha^{j}, \quad \text { and } \alpha^{j}=\left\langle\alpha, e_{j}\right\rangle .
$$

The operators $T_{j}, j=1,2, \ldots, d$, can also be written in the following form

$$
\begin{aligned}
T_{j} f(x) & =\frac{\partial}{\partial x_{j}} f(x)+\sum_{i=1}^{d} \frac{k\left(\alpha_{i}\right) \alpha_{i}^{j}}{1-e^{-\left\langle\alpha_{i}, x\right\rangle}}\left\{f(x)-f\left(r_{\alpha_{i}} x\right\}\right. \\
& +2 \sum_{i=1}^{d} \frac{k\left(2 \alpha_{i}\right) \alpha_{i}^{j}}{1-e^{-2\left\langle\alpha_{i}, x\right\rangle}}\left\{f(x)-f\left(r_{2 \alpha_{i}} x\right)\right\} \\
& -\frac{1}{2}\left(\sum_{i=1}^{d}\left(k\left(\alpha_{i}\right)+2 k\left(2 \alpha_{i}\right)\right) \alpha_{i}^{j}\right) f(x),
\end{aligned}
$$

by using the fact that from (2.3) we have

$$
\forall i \in\{1,2, \ldots, d\}, r_{2 \alpha_{i}} x=r_{\alpha_{i}} x,
$$

we obtain

$$
\begin{aligned}
T_{j} f(x)=\frac{\partial}{\partial x_{j}} f(x) & +\sum_{i=1}^{d}\left[\frac{k\left(\alpha_{i}\right)}{1-e^{-\left\langle\alpha_{i}, x\right\rangle}}+\frac{2 k\left(2 \alpha_{i}\right)}{1-e^{-2\left\langle\alpha_{i}, x\right\rangle}}\right] \alpha_{i}^{j}\left\{f(x)-f\left(r_{\alpha_{i}} x\right)\right\} \\
& -\frac{1}{2}\left(\sum_{i=1}^{d}\left(k\left(\alpha_{i}\right)+2 k\left(2 \alpha_{i}\right)\right) \alpha_{i}^{j}\right) f(x) .
\end{aligned}
$$




\subsection{The Eigenfunctions of the Cherednik Operators}

We denote by $G_{\lambda}, \lambda \in \mathbb{C}^{d}$, the eigenfunction of the operators $T_{j}, j=1,2, \ldots d$. It is the unique analytic function on $\mathbb{R}^{d}$ which satisfies the differential difference system

$$
\begin{cases}T_{j} G_{\lambda}(x) & =-i \lambda_{j} G_{\lambda}(x), x \in \mathbb{R}^{d}, j=1,2, \ldots, d, \\ G_{\lambda}(0) & =1 .\end{cases}
$$

It is called the Opdam-Cherednik kernel.

We consider the function $F_{\lambda}, \lambda \in \mathbb{C}^{d}$ defined by

$$
\forall x \in \mathbb{R}^{d}, F_{\lambda}(x)=\frac{1}{|W|} \sum_{w \in W} G_{\lambda}(w x) .
$$

This function is the unique analytic $W$-invariant function on $\mathbb{R}^{d}$, which satisfies the partial differential equation

$$
\begin{cases}p(T) F_{\lambda}(x) & =p(-i \lambda) F_{\lambda}(x), \quad x \in \mathbb{R}^{d} \\ F_{\lambda}(0) & =1\end{cases}
$$

for all $W$-invariant polynomial $p$ on $\mathbb{R}^{d}$ and $p(T)=p\left(T_{1}, T_{2}, \ldots, T_{d}\right)$. It is called the Heckman-Opdam kernel.

The functions $G_{\lambda}$ and $F_{\lambda}$ possess the following properties:

i) - For all $\lambda \in \mathbb{C}^{d}$ the fonction $x \rightarrow G_{\lambda}(x)$ is of class $C^{\infty}$ on $\mathbb{R}^{d}$.

- For all $x \in \mathbb{R}^{d}$ the function $\lambda \rightarrow G_{\lambda}(x)$ is entire on $\mathbb{C}^{d}$.

ii) We have

$$
\forall x \in \mathbb{R}^{d}, \forall \lambda \in \mathbb{C}^{d}, \overline{G_{\lambda}(x)}=G_{-\bar{\lambda}}(x) .
$$

iii) We have

$$
\forall x \in \mathbb{R}^{d}, \forall \lambda \in \mathbb{C}^{d},\left|G_{\lambda}(x)\right| \leq G_{i \operatorname{Im}(\lambda)}(x) .
$$

iv) We have

$$
\begin{aligned}
& \forall x \in \mathbb{R}^{d}, \forall \lambda \in \mathbb{R}^{d},\left|G_{\lambda}(x)\right| \leq|W|^{1 / 2} . \\
& \forall x \in \mathbb{R}^{d}, \forall \lambda \in \mathbb{R}^{d},\left|F_{\lambda}(x)\right| \leq|W|^{1 / 2} .
\end{aligned}
$$

v) The function $F_{0}$ satisfies the estimate

$$
\forall x \in \overline{\mathfrak{a}_{+}}, F_{0}(x) \asymp e^{-\langle\rho, x\rangle} \prod_{i=1}^{d}\left(1+\left\langle\alpha_{i}, x\right\rangle\right) .
$$


vi) Let $p$ and $q$ be polynomials of degree $m$ and $n$. Then there exists a positive constant $M$ such that for all $x \in \mathbb{R}^{d}$ and $\lambda \in \mathbb{C}^{d}$, we have

$$
\left|p\left(\frac{\partial}{\partial \lambda}\right) q\left(\frac{\partial}{\partial x}\right) G_{\lambda}(x)\right| \leq M(1+\|\lambda\|)^{n}(1+\|x\|)^{m} F_{0}(x) e^{\max _{w \in W} \operatorname{Im}\langle w \lambda, x\rangle} .
$$

vii) The function $G_{\lambda}, \lambda \in \mathbb{C}^{d}$, admits the following Laplace type representation

$$
\forall x \in \mathbb{R}^{d}, G_{\lambda}(x)=\left\langle K_{x}, e^{-i\langle\lambda, .\rangle}\right\rangle,
$$

where $K_{x}$ is some distribution in $\mathcal{E}^{\prime}\left(\mathbb{R}^{d}\right)$ (the space of distributions on $\mathbb{R}^{d}$ with compact support) with support in $\Gamma=\operatorname{conv}\{w x, w \in W\}$ (the convexe hull of the orbit of $x$ under $W$ ).

viii) From (2.13), (2.21) we deduce that the function $F_{\lambda}, \lambda \in \mathbb{C}^{d}$, possesses the Laplace type representation

$$
\forall x \in \mathbb{R}^{d}, F_{\lambda}(x)=\left\langle K_{x}^{W}, e^{-i\langle\lambda, .\rangle}\right\rangle,
$$

where $K_{x}^{W}$ is the distribution in $\mathcal{E}^{\prime}\left(\mathbb{R}^{d}\right)$ with support in $\Gamma$ given by

$$
K_{x}^{W}=\frac{1}{|W|} \sum_{w \in W} K_{w x}
$$

\section{The Transmutation Operator $V_{k}$ and its Dual ${ }^{t} V_{k}$ Associated with the Cherednik Operators (see [9])}

Notations. We denote by

- $\mathcal{E}\left(\mathbb{R}^{d}\right)$ the space of $C^{\infty}$-functions on $\mathbb{R}^{d}$. Its topology is defined by the semi-norms

$$
q_{n, K}(\varphi)=\sup _{\substack{|\mu| \leq n \\ x \in K}}\left|D^{\mu} \varphi(x)\right|,
$$

where $K$ is a compact of $\mathbb{R}^{d}, n \in \mathbb{N}$, and

$$
D^{\mu}=\frac{\partial^{|\mu|}}{\partial^{\mu_{1}} x_{1} \partial^{\mu_{2}} x_{2} \ldots \partial^{\mu_{d}} x_{d}}, \mu \in \mathbb{N}^{d},|\mu|=\sum_{i=1}^{d} \mu_{i} .
$$

- $\mathcal{D}\left(\mathbb{R}^{d}\right)$ the space of $C^{\infty}$-functions on $\mathbb{R}^{d}$ with compact support. We have

$$
\mathcal{D}\left(\mathbb{R}^{d}\right)=\bigcup_{a>0} \mathcal{D}_{a}\left(\mathbb{R}^{d}\right)
$$


where $\mathcal{D}_{a}\left(\mathbb{R}^{d}\right)$ is the space of $C^{\infty}$-functions on $\mathbb{R}^{d}$ with support in the closed ball $B(0, a)$ of center 0 and radius $a$. The topology of $\mathcal{D}_{a}\left(\mathbb{R}^{d}\right)$ is defined by the semi-norms

$$
p_{n}(\psi)=\sup _{\substack{|\mu| \geq n \\ x \in B(0, a)}}\left|D^{\mu} \psi(x)\right|, \quad n \in \mathbb{N} .
$$

The space $\mathcal{D}\left(\mathbb{R}^{d}\right)$ is equipped with the inductive limit topology.

By using the distribution $K_{x}, x \in \mathbb{R}^{d}$, given by (2.21), we define the transmutation operator called also the trigonometric Dunkl intertwining operator $V_{k}$ on $\mathcal{E}\left(\mathbb{R}^{d}\right)$ by

$$
\forall x \in \mathbb{R}^{d}, V_{k}(g)(x)=\left\langle K_{x}, g\right\rangle .
$$

The operator $V_{k}$ is the unique linear topological isomorphism from $\mathcal{E}\left(\mathbb{R}^{d}\right)$ onto itself satisfying the transmutation relations

$$
\forall x \in \mathbb{R}^{d}, T_{j} V_{k}(g)(x)=V_{k}\left(\frac{\partial}{\partial y_{j}} g\right)(x), j=1,2, \ldots, d,
$$

and the condition

$$
V_{k}(g)(0)=g(0) \text {. }
$$

The dual ${ }^{t} V_{k}$ of the operator $V_{k}$ is defined by the following duality relation

$$
\int_{\mathbb{R}^{d}}{ }^{t} V_{k}(f)(y) g(y) d y=\int_{\mathbb{R}^{d}} V_{k}(g)(x) f(x) \mathcal{A}_{k}(x) d x,
$$

with $f$ in $\mathcal{D}\left(\mathbb{R}^{d}\right)$ and $g$ in $\mathcal{E}\left(\mathbb{R}^{d}\right)$.

The operator ${ }^{t} V_{k}$ is a linear topological isomorphism from $\mathcal{D}\left(\mathbb{R}^{d}\right)$ onto itself satisfying the transmutation relations

$$
\forall y \in \mathbb{R}^{d},{ }^{t} V_{k}\left(\left(T_{j}+S_{j}\right) f\right)(y)=\frac{\partial}{\partial y_{j}}{ }^{t} V_{k}(f)(y), \quad j=1,2, \ldots, d,
$$

where $S_{j}$ is the operator on $\mathcal{D}\left(\mathbb{R}^{d}\right)$ given by

$$
\forall x \in \mathbb{R}^{d}, S_{j}(h)(x)=\sum_{\alpha \in \mathcal{R}_{+}} k(\alpha) \alpha^{j} h\left(r_{\alpha} x\right) .
$$

Remark 3.1. By using the distribution $K_{x}^{W}$ given by (2.23) we have defined and studied in [12] the transmutation operator called also the trigonometric Dunkl intertwining operator $V_{k}^{W}$ on $\mathcal{E}\left(\mathbb{R}^{d}\right)^{W}$ (the subspace of functions of $\mathcal{E}\left(\mathbb{R}^{d}\right.$ ) which are $W$-invariant), and we have studied also its dual ${ }^{t} V_{k}^{W}$ on $\mathcal{D}\left(\mathbb{R}^{d}\right)^{W}$ 
(the subspace of functions of $\mathcal{D}\left(\mathbb{R}^{d}\right)$ which are $W$-invariant), and we have given properties of these operators.

Proposition 3.2. Let $t>0$. For all $x, y \in \mathbb{R}^{d}$, we have

$$
V_{k}\left(p_{t}(u, .)\right)(x)=\int_{\mathbb{R}^{d}} e^{-t\|\lambda\|^{2}} G_{\lambda}(x) e^{i\langle\lambda, u\rangle} d \lambda,
$$

where $p_{t}(u, z)$ is the classical heat kernel given by

$$
\forall u, z \in \mathbb{R}^{d}, p_{t}(u, z)=\int_{\mathbb{R}^{d}} e^{-t\|\lambda\|^{2}} e^{i\langle\lambda, u-z\rangle} d \lambda=\left(\frac{\pi}{t}\right)^{d / 2} e^{-\frac{\|u-z\|^{2}}{4 t}} .
$$

Proof. From (3.1) we have

$$
\forall x \in \mathbb{R}^{d} \backslash\{0\}, u \in \mathbb{R}^{d}, V_{k}\left(p_{t}(u, .)\right)(x)=\left\langle K_{x}, p_{t}(u, .)\right\rangle .
$$

Thus from (3.8), (3.7), for all $x \in \mathbb{R}^{d} \backslash\{0\}, u \in \mathbb{R}^{d}$, we have

$$
V_{k}\left(p_{t}(u, .)\right)(x)=\left\langle K_{x}(z), \int_{\mathbb{R}^{d}} e^{-t\|\lambda\|^{2}} e^{i\langle\lambda, u-z\rangle} d \lambda\right\rangle .
$$

As the distribution $K_{x}$ belongs to $\mathcal{E}^{\prime}\left(\mathbb{R}^{d}\right)$, then the relation (3.9) can also be written in the form

$$
V_{k}\left(p_{t}(u, .)\right)(x)=\int_{\mathbb{R}^{d}} e^{-t\|\lambda\|^{2}}\left\langle K_{x}(z), e^{-i\langle\lambda, z\rangle}\right\rangle e^{i\langle\lambda, u\rangle} d \lambda .
$$

Thus from, (3.10), (2.21), for all $x \in \mathbb{R}^{d} \backslash\{0\}, u \in \mathbb{R}^{d}$, we get

$$
V_{k}\left(p_{t}(u, .)\right)(x)=\int_{\mathbb{R}^{d}} e^{-t\|\lambda\|^{2}} G_{\lambda}(x) e^{i\langle\lambda, u\rangle} d \lambda .
$$

On the other hand from (3.3), (3.7), (2.12), for all $u \in \mathbb{R}^{d}$, we have

$$
V_{k}\left(p_{t}(u, .)\right)(0)=p_{t}(u, 0)=\int_{\mathbb{R}^{d}} e^{-t\|\lambda\|^{2}} e^{i\langle\lambda, u\rangle} d \lambda .
$$

We deduce (3.6) from (3.11), (3.12) and the continuity of the function $x \rightarrow$ $V_{k}\left(p_{t}(u,).\right)(x)$ at $x=0$.

Proposition 3.3. Let $t>0$. The function $V_{k}\left(p_{t}(u,).\right)(x)$ is of class $C^{\infty}$ on $\mathbb{R}^{d} \times \mathbb{R}^{d}$ with respect to the variables $x$ and $u$ and satisfies the equation

$$
\forall x, u \in \mathbb{R}^{d},\left(T_{j}+\frac{\partial}{\partial u_{j}}\right) V_{k}\left(p_{t}(u, .)\right)(x)=0, j=1,2, \ldots, d .
$$


Proof. We obtain the results by derivation under the integral sign with respect to the variables $x_{j}, u_{j}, j=1,2, \ldots, d$, in the relation (3.6) by using (2.20), (2.18), and by applying the relation (2.12).

\section{Proposition 3.4.}

i) Let $t>0$. There exists a positive function $M_{0}(t)$ such that

$$
\forall x, u \in \mathbb{R}^{d},\left|V_{k}\left(p_{t}(u, .)\right)(x)\right| \leq M_{0}(t) F_{0}(x) .
$$

ii) Let $t>0$. We have

$$
\forall x \in \mathbb{R}^{d}, \quad \lim _{\|u\| \rightarrow+\infty} V_{k}\left(p_{t}(u, .)\right)(x)=0 .
$$

iii) Let $t>0$. The function $(x, u) \rightarrow V_{k}\left(p_{t}(u,).\right)(x)$ is bounded on $\mathbb{R}^{d} \times \mathbb{R}^{d}$ and we have

$$
\lim _{\|(x, u)\| \rightarrow+\infty} V_{k}\left(p_{t}(u, .)\right)(x)=0 .
$$

\section{Proof.}

i) We deduce (3.14) from (3.6), (2.20), (2.18).

ii) By using (3.6) and the fact that from (2.17) the function $\lambda \rightarrow e^{-t\|\lambda\|^{2}} G_{\lambda}(x)$ is for all $x \in \mathbb{R}^{d}$, integrable with respect to the Lebesgue measure on $\mathbb{R}^{d}$, we deduce (3.15) from Riemann-Lebesgue Lemma.

iii) - The relations (3.14), (2.18) imply that the function $(x, u) \rightarrow$ $V_{k}\left(p_{t}(u,).\right)(x)$ is bounded on $\mathbb{R}^{d} \times \mathbb{R}^{d}$.

- We consider $x \in \mathbb{R}_{r e g}^{d}=\mathbb{R}^{d} \backslash \cup_{i=1}^{d} H_{\alpha_{i}}$. There exists $w \in W$ such that $x=w x^{+}$with $x^{+} \in \mathfrak{a}_{+}$. Then from (3.14), (2.19) there exists a positive function $M_{1}(t)$ such that

$$
\forall x \in \mathbb{R}_{r e g}^{d}, u \in \mathbb{R}^{d},\left|V_{k}\left(p_{t}(u, .)\right)(x)\right| \leq M_{1}(t)\left(\prod_{i=1}^{d}\left(1+\left\langle\alpha_{i}, x^{+}\right\rangle\right)\right) e^{-\left\langle\rho, x^{+}\right\rangle} .
$$

Thus the function $V_{k}\left(p_{t}(u,).\right)(x)$ tends to zero when $x$ goes to infinity, uniformly with respect to the variable $u$. We deduce (3.16) from this result and the continuity of the function $V_{k}\left(p_{t}(u,).\right)(x)$ on $\mathbb{R}^{d}$ with respect to the variable $x$. 


\section{Positivity of the Operators $V_{k}$ and ${ }^{t} V_{k}$}

In this section we prove first that for $t>0$, the function $(x, u) \rightarrow V_{k}\left(p_{t}(u,).\right)(x)$ given by (3.6) is positive on $\mathbb{R}^{d} \times \mathbb{R}^{d}$, and next we deduce the positivity of the operators $V_{k}$ and ${ }^{t} V_{k}$.

\section{Proposition 4.1.}

i) Let $t>0$. For all $x, u \in \mathbb{R}^{d}$ the function $V_{k}\left(p_{t}(u,).\right)(x)$ is real.

ii) Let $t>0$. The function $V_{k}\left(p_{t}(u,).\right)(x)$ is strictly positive on the set

$$
Y=\left\{(x, u) \in \mathbb{R}^{d} \times \mathbb{R}^{d} ; \quad x=0, u \in \mathbb{R}^{d}\right\}
$$

\section{Proof.}

i) We obtain the result from the relations (3.6), (2.15) and a change of variables.

ii) By using the relation

$$
\forall \lambda \in \mathbb{R}^{d}, G_{\lambda}(0)=1,
$$

we deduce from (3.6), (3.3), (3.7) that

$$
\forall u \in \mathbb{R}^{d}, V_{k}\left(p_{t}(u, .)\right)(0)=p_{t}(u, 0)=\left(\frac{\pi}{t}\right)^{d / 2} e^{-\frac{\|u\|^{2}}{4 t}}>0 .
$$

Proposition 4.2. Let $t>0$. We consider the function $U_{t}(x, u)$ defined by

$$
\forall x, u \in \mathbb{R}^{d}, U_{t}(x, u)=V_{k}\left(p_{t}(u, .)\right)(x) .
$$

For all $\alpha_{i}, i \in\{1,2, \ldots, d\}$, and $x, u \in \mathbb{R}^{d}$, we have

$$
\begin{aligned}
U_{t}\left(r_{\alpha_{i}} x, u\right)-U_{t}(x, u) & =-\left\langle\breve{\alpha}_{i}, x\right\rangle\left\langle\nabla U_{t}(x, u), \alpha_{i}\right\rangle \\
& +\frac{1}{2}\left(\left\langle\breve{\alpha}_{i}, x\right\rangle\right)^{2} \alpha_{i}^{t} D^{2} U_{t}(\xi, u) \alpha_{i}
\end{aligned}
$$

with some $\xi$ on the line segment between $x$ and $r_{\alpha_{i}} x$.

Proof. We obtain (4.2) from the relation (2.4) and Taylor's formula.

Proposition 4.3. There exists $j_{0} \in\{1,2, \ldots, d\}$ such that

$$
\rho_{j_{0}}=\frac{1}{2} \sum_{i=1}^{d}\left(k\left(\alpha_{i}\right)+2 k\left(2 \alpha_{i}\right)\right) \alpha_{i}^{j_{0}} \neq 0
$$


Proof. We denote by

$$
K_{i}=\frac{1}{2}\left(k\left(\alpha_{i}\right)+2 k\left(2 \alpha_{i}\right)\right)
$$

and we consider the matrix $M$ given by

$$
M=\left(\begin{array}{llll}
K_{1} \alpha_{1}^{1} & K_{2} \alpha_{2}^{1} & \ldots & K_{d} \alpha_{d}^{1} \\
K_{1} \alpha_{1}^{2} & K_{2} \alpha_{2}^{2} & \ldots & K_{d} \alpha_{d}^{2} \\
\cdots & \cdots & \cdots & \cdots \\
K_{1} \alpha_{1}^{d} & K_{2} \alpha_{2}^{d} & \cdots & K_{d} \alpha_{d}^{d}
\end{array}\right)
$$

We have

$$
\operatorname{det}(M)=\left(\prod_{i=1}^{d} K_{i}\right) \operatorname{det}\left(\left(\alpha_{i}^{j}\right)_{1 \leq i, j \leq d}\right)
$$

On the other hand the $\operatorname{det}(M)$ can also be written in the following form

$$
\operatorname{det}(M)=\left|\begin{array}{lllll}
\left(\sum_{i=1}^{d}\right. & \left.K_{i} \alpha_{i}^{1}\right) & K_{2} \alpha_{2}^{1} & \ldots & K_{d} \alpha_{d}^{1} \\
\left(\sum_{i=1}^{d}\right. & \left.K_{i} \alpha_{i}^{2}\right) & K_{2} \alpha_{2}^{2} & \ldots & K_{d} \alpha_{d}^{2} \\
\cdots & \ldots & \ldots & \ldots & \ldots \\
\left(\sum_{i=1}^{d}\right. & \left.K_{i} \alpha_{i}^{d}\right) & K_{2} \alpha_{2}^{d} & \ldots & K_{d} \alpha_{d}^{d}
\end{array}\right|=\left|\begin{array}{llll}
\rho_{1} & K_{2} \alpha_{2}^{1} & \ldots & K_{d} \alpha_{d}^{1} \\
\rho_{2} & K_{2} \alpha_{2}^{2} & \ldots & K_{d} \alpha_{d}^{2} \\
\cdots & \ldots & \ldots & \ldots \\
\rho_{d} & K_{2} \alpha_{2}^{d} & \ldots & K_{d} \alpha_{d}^{d}
\end{array}\right|
$$

From (2.8) and the fact that the vectors $\alpha_{1}, \alpha_{2}, \ldots \alpha_{d}$ are linearly independent, the relation (4.4) implies that $\operatorname{det}(M) \neq 0$. Then we obtain (4.3) from the relation $(4.5)$.

Notations. We denote by:

- $C_{\ell}, \ell=1,2, \ldots, 2 d$, the Weyl chambers relating to the root system $\mathcal{R}$, with $C_{1}=\mathfrak{a}_{+}$.

- $\overline{C_{\ell}}, \ell=1,2, \ldots, 2 d$, the closure of $C_{\ell}, \ell=1,2, \ldots, 2 d$. We have $\mathbb{R}^{d}=\cup_{\ell=1}^{2 d} \overline{C_{\ell}}$.

- $\partial \overline{C_{\ell}}, \ell=1,2, \ldots, 2 d$, the border of $\overline{C_{\ell}}, \ell=1,2, \ldots, 2 d$.

Theorem 4.4. For all $t>0$, we have

$$
\forall x, u \in \mathbb{R}^{d}, V_{k}\left(p_{t}(u, .)\right)(x) \geq 0 .
$$

Proof. The proof is made up of two parts.

In the first part we obtain some results concerning the positivity of the function $U_{t}(x, u)$ given by (4.1) on each of the sets $\overline{C_{\ell}} \times \mathbb{R}^{d}, \ell=1,2, \ldots, 2 d$.

In the second part we use the fact that $\mathbb{R}^{d} \times \mathbb{R}^{d}=\left(\cup_{\ell=1}^{2 d}\right) \overline{C_{\ell}} \times \mathbb{R}^{d}$ and the result of the first part to deduce the positivity of the function $U_{t}(x, u)$ on $\mathbb{R}^{d} \times \mathbb{R}^{d}$. 
1st Part. We consider the set $Y_{\ell}$ defined by

$$
Y_{\ell}=\left\{(x, u) \in \mathbb{R}^{d} \times \mathbb{R}^{d} ; \quad x \in \overline{C_{\ell}}, u \in \mathbb{R}^{d}\right\} .
$$

We denote by

$$
\mathcal{V}_{t}^{\ell}(x, u)=U_{t}(x, u) 1_{Y_{\ell}}(x, u),
$$

where $1_{Y_{\ell}}$ is the characteristic function of the set $Y_{\ell}$. From Proposition 4.1 ii), the function $\mathcal{V}_{t}^{\ell}(x, u)$ is strictly positive on the set $Y$. We shall prove that it is positive on the set $Y_{\ell} \backslash Y$. If not we suppose by using Proposition $4.1 \mathrm{i}$ ) and Proposition 3.4 iii) that it attains a strictly negative absolute minimum at $\left(x^{\ell}, u^{\ell}\right) \in Y_{\ell} \backslash Y \quad$ i.e.

$$
\mathcal{V}_{t}^{\ell}\left(x^{\ell}, u^{\ell}\right)=\inf _{(x, u) \in Y_{\ell}} \mathcal{V}_{t}^{\ell}(x, u)<0
$$

There are two possibilities: The point $\left(x^{\ell}, u^{\ell}\right)$ is in the open subset $\left(Y_{\ell} \backslash Y\right)^{0}$ of the set $Y_{\ell} \backslash Y$, or in the set

$$
Y_{\ell}^{0}=\left\{(x, u) \in \mathbb{R}^{d} \times \mathbb{R}^{d} ; \quad x \in \partial \overline{C_{\ell}}, u \in \mathbb{R}^{d}\right\} .
$$

We suppose that $\left(x^{\ell}, u^{\ell}\right) \in\left(Y_{\ell} \backslash Y\right)^{0}$. As the point $\left(x^{\ell}, u^{\ell}\right)$ is an absolute minimum, then we have

$$
\frac{\partial}{\partial x_{j}} \mathcal{V}_{t}^{\ell}\left(x^{\ell}, u^{\ell}\right)=\frac{\partial}{\partial u_{j}} \mathcal{V}_{t}^{\ell}\left(x^{\ell}, u^{\ell}\right)=0, \quad j=1,2, \ldots, d .
$$

By using the fact that

$$
\forall i \in\{1,2, \ldots, d\},\left(r_{\alpha_{i}} x^{\ell}, u^{\ell}\right) \notin Y_{\ell},
$$

and by applying the relation (4.1), (3.13), (2.11), (4.9), (4.7), we obtain the linear homogeneous system

$$
\left.\sum_{i=1}^{d}\left[k\left(\alpha_{i}\right)\left(\frac{1}{1-e^{-\left\langle\alpha_{i}, x^{\ell}\right\rangle}}-\frac{1}{2}\right)+2 k\left(2 \alpha_{i}\right)\left(\frac{1}{1-e^{-2\left\langle\alpha_{i}, x^{\ell}\right\rangle}}-\frac{1}{2}\right)\right)\right] \alpha_{i}^{j}=0,
$$

which can also be written in the form

$$
j=1,2, \ldots, d,
$$

$$
\sum_{i=1}^{d} \alpha_{i}^{j} X_{i}=0, \quad j=1,2, \ldots, d,
$$


with

$$
X_{i}=\left(k\left(\alpha_{i}\right)+k\left(2 \alpha_{i}\right)\right) \operatorname{coth}\left\langle\frac{\alpha_{i}}{2}, x^{\ell}\right\rangle+k\left(2 \alpha_{i}\right) \tanh \left\langle\frac{\alpha_{i}}{2}, x^{\ell}\right\rangle, i=1,2, \ldots, d .
$$

We write the linear homogenous system (4.10) in the form of the matrix equation

$$
B \chi=0,
$$

where $B=\left(\alpha_{i}^{j}\right)_{1 \leq i, j \leq d}$ is the $d \times d$ matrix of coefficients, $\chi$ the column matrix of elements $X_{1}, X_{2}, \ldots, X_{d}$ and the zero in the right member denotes the zero $d \times 1$ matrix. As the vectors $\alpha_{1}, \alpha_{2}, \ldots, \alpha_{d}$ are linearly independent then we have

$$
\operatorname{det}(B) \neq 0 \text {. }
$$

Thus

$$
\chi=0 \Leftrightarrow \forall i \in\{1,2, \ldots, d\}, X_{i}=0 .
$$

On the other hand from (4.11), (2.8), and the fact that $\left(x^{\ell}, u^{\ell}\right) \in\left(Y_{\ell} \backslash Y\right)^{0}$, implies that for all $i \in\{1,2, \ldots, d\},\left\langle\alpha_{i}, x^{\ell}\right\rangle \neq 0$, we deduce that

$$
\forall i \in\{1,2, \ldots, d\}, X_{i} \neq 0 .
$$

Then from (4.14), (4.15) we obtain a contradiction and then the point $\left(x^{\ell}, u^{\ell}\right)$ does not belongs to the set $\left(Y_{\ell} \backslash Y\right)^{0}$. Then it is in the set $Y_{\ell}^{0}$.

2nd Part. From Proposition 4.1 ii), the function $U_{t}(x, u)$ given by (4.1) is strictly positive on the set $Y$. We shall prove that it is positive on the set $\mathbb{R}^{d} \times \mathbb{R}^{d} \backslash Y$. If not we suppose by using Proposition 4.1.i) and Proposition 3.4.iii) that it attains a strictly negative absolute minimum at $\left(x_{0}, u_{0}\right) \in \mathbb{R}^{d} \times \mathbb{R}^{d} \backslash Y$.

We suppose first that the point $\left(x_{0}, u_{0}\right)$ is in the subset $Y_{1} \backslash Y$. As we have

$$
U_{t}\left(x_{0}, u_{0}\right)=\inf _{(x, u) \in \mathbb{R}^{d} \times \mathbb{R}^{d}} U_{t}(x, u) \leq \inf _{(x, u) \in Y_{1}} U_{t}(x, u),
$$

then the point $\left(x_{0}, u_{0}\right)$ is a strictly negative absolute minimum in $Y_{1} \backslash Y$. By using the result of the first part, we deduce that the point $\left(x_{0}, u_{0}\right)$ is not in the open subset $\left(Y_{1} \backslash Y\right)^{0}$ but it belongs to $Y_{1}^{0}$ given by (4.8).

Next we make the same previous suppository with each of the sets $Y_{2} \backslash Y, \ldots$, $Y_{d} \backslash Y$, and we show that the point $\left(x_{0}, u_{0}\right)$ does not belongs to each of the open subsets $\left(Y_{2} \backslash Y\right)^{0}, \ldots,\left(Y_{d} \backslash Y\right)^{0}$, and it is in each of the sets $Y_{2}^{0}, \ldots, Y_{d}^{0}$. Then $\left(x_{0}, u_{0}\right) \in Y^{0}=\cup_{\ell=1}^{2 d} Y_{\ell}^{0}$.

But we have

$$
Y^{0}=\left\{(x, u) \in \mathbb{R}^{d} \times \mathbb{R}^{d} ; \forall i \in\{1,2, \ldots, d\},\left\langle\alpha_{i}, x\right\rangle=0, u \in \mathbb{R}^{d}\right\} .
$$


As the point $x=0$ belongs to the set $\left\{x \in \mathbb{R}^{d} ; \forall i \in\{1,2, \ldots, d\},\left\langle\alpha_{i}, x\right\rangle=0\right\}$, and the vectors $\alpha_{1}, \alpha_{2}, \ldots, \alpha_{d}$ are linearly independent then

$$
Y^{0}=\left\{(x, u) \in \mathbb{R}^{d} \times \mathbb{R}^{d} ; x=0, u \in \mathbb{R}^{d}\right\} .
$$

Thus $x_{0}=0$. We shall prove in the following that the point $\left(x_{0}, u_{0}\right)$ is not in the set $Y^{0}$. As the point $\left(x_{0}, u_{0}\right)$ is a strictly negative absolute minimum, then we have the following relations

$$
U_{t}\left(x_{0}, u_{0}\right)=\inf _{(x, u) \in \mathbb{R}^{d} \times \mathbb{R}^{d}} U_{t}(x, u)<0,
$$

and

$$
\frac{\partial}{\partial x_{j}} U_{t}\left(x_{0}, u_{0}\right)=\frac{\partial}{\partial u_{j}} U_{t}\left(x_{0}, u_{0}\right)=0, j=1,2, \ldots, d .
$$

We consider the relations (4.1), (3.13), (2.11) and we write them for $x \in \mathbb{R}^{d}$, $u_{0}$, and for $j_{0}$ of Proposition 4.3, and we obtain

$$
\begin{aligned}
\frac{\partial}{\partial x_{j_{0}}} U_{t}\left(x, u_{0}\right) & +\frac{\partial}{\partial u_{j_{0}}} U_{t}\left(x, u_{0}\right)+\sum_{i=1}^{d}\left[\frac{k\left(\alpha_{i}\right)}{1-e^{-\left\langle\alpha_{i}, x\right\rangle}}+\frac{2 k\left(2 \alpha_{i}\right)}{1-e^{-2\left\langle\alpha_{i}, x\right\rangle}}\right] \alpha_{i}^{j_{0}} \\
& \times\left\{U_{t}\left(x, u_{0}\right)-U_{t}\left(r_{\alpha_{i}} x, u_{0}\right)\right\}=\rho_{j_{0}} U_{t}\left(x, u_{0}\right) .
\end{aligned}
$$

Then by passing to the limit in (4.18) when $\left\langle\alpha_{i}, x\right\rangle$, for all $i \in\{1,2, \ldots, d\}$, goes to $\left\langle\alpha_{i}, x_{0}\right\rangle=0$, and by using Proposition 4.2 , and the relation (4.17), we obtain

$$
\rho_{j_{0}} U_{t}\left(x_{0}, u_{0}\right)=0 .
$$

But from (4.3) we have $\rho_{j_{0}} \neq 0$. Then

$$
U_{t}\left(x_{0}, u_{0}\right)=0 .
$$

Thus (4.16) and (4.19) imply a contradiction and the point $\left(x_{0}, u_{0}\right)$ is not in the set $Y^{0}$. Then the function $U_{t}(x, u)$ is positive on the set $\mathbb{R}^{d} \times \mathbb{R}^{d} \backslash Y$. We deduce (4.6) from this result and the fact that the function $V_{k}\left(p_{t}(u,).\right)(x)$ is positive on the set $Y$.

Theorem 4.5. For all positive function $f$ in $\mathcal{D}\left(\mathbb{R}^{d}\right)$, we have

$$
\forall y \in \mathbb{R}^{d},{ }^{t} V_{k}(f)(y) \geq 0 .
$$


Proof. From the relations (3.4), (3.7), for all $t>0$ and $y \in \mathbb{R}^{d}$, we have

$$
\int_{\mathbb{R}^{d}}{ }^{t} V_{k}(f)(x) p_{t}(y, x) d x=\int_{\mathbb{R}^{d}} f(z) V_{k}\left(p_{t}(y, .)\right)(z) \mathcal{A}_{k}(z) d z .
$$

But from Theorem 4.4, the second member of this relation is positive. Then

$$
\int_{\mathbb{R}^{d}}{ }^{t} V_{k}(f)(x) p_{t}(y, x) d x={ }^{t} V_{k}(f) * E_{t}(y) \geq 0,
$$

with $E_{t}$ is the classical Gauss kernel given by

$$
\forall u \in \mathbb{R}^{d}, E_{t}(u)=\int_{\mathbb{R}^{d}} e^{-t\|\lambda\|^{2}} e^{i\langle\lambda, u\rangle} d \lambda,
$$

and $*$ is the classical convolution product on $\mathbb{R}^{d}$.

Thus

$$
{ }^{t} V_{k}(f)(y)=\lim _{t \rightarrow 0}{ }^{t} V_{k}(f) * E_{t}(y) \geq 0
$$

Theorem 4.6. There exists a $\sigma$-algebra $\mathfrak{m}$ in $\mathbb{R}^{d}$ which contains all Borel sets in $\mathbb{R}^{d}$, and for each $y \in \mathbb{R}^{d}$, there exists a unique positive measure $\nu_{y}$ on $\mathfrak{m}$ such that for every $f$ in $\mathcal{D}\left(\mathbb{R}^{d}\right)$, we have

$$
{ }^{t} V_{k}(f)(y)=\int_{\mathbb{R}^{d}} f(x) d \nu_{y}(x) .
$$

The measure $\nu_{y}$ satisfies

$$
\nu_{y}(K)<+\infty, \text { for every compact } K \subset \mathbb{R}^{d} .
$$

Proof. We deduce the results from the relation (4.18) and Theorem 2.14, p. 42 of $[6]$.

Theorem 4.7. For all $g$ in $\mathcal{E}\left(\mathbb{R}^{d}\right)$, positive, we have

$$
\forall x \in \mathbb{R}^{d}, \quad V_{k}(g)(x) \geq 0 .
$$

Proof. From the relation (3.4) for all $f$ in $\mathcal{D}\left(\mathbb{R}^{d}\right)$ positive, and $g$ in $\mathcal{E}\left(\mathbb{R}^{d}\right)$ positive we have

$$
\int_{\mathbb{R}^{d}} V_{k}(g)(x) f(x) \mathcal{A}_{k}(x) d x=\int_{\mathbb{R}^{d}}{ }^{t} V_{k}(f)(y) g(y) d y .
$$


By applying Theorem 4.5 to the second member, we deduce that

$$
\int_{\mathbb{R}^{d}} f(x) V_{k}(g)(x) \mathcal{A}_{k}(x) d x=\left\langle\mathcal{T}_{V_{k}(g) \mathcal{A}_{k}} f\right\rangle
$$

where $\mathcal{T}_{V_{k}(g)_{\mathcal{A}_{k}}}$ is the distribution of $\mathcal{D}^{\prime}\left(\mathbb{R}^{d}\right)$ (the space of distributions on $\mathbb{R}^{d}$ ) given by the function $V_{k}(g) \mathcal{A}_{k}$. From (4.22) and Theorem $\mathrm{V}$ of [8], p. 29, this distribution is the positive measure of density $V_{k}(g) \mathcal{A}_{k}$ with respect to the Lebesgue measure on $\mathbb{R}^{d}$. Then by using (2.7) and the continuity of the function $V_{k}(g)$ on $\mathbb{R}^{d}$, we obtain (4.21).

Theorem 4.8. There exists a $\sigma$-algebra $\mathfrak{m}$ in $\mathbb{R}^{d}$ which contains all Borel sets in $\mathbb{R}^{d}$ and for each $x \in \mathbb{R}^{d}$, there exists a unique positive measure $\mu_{x}$ on $\mathfrak{m}$ with support in the closed ball $B(0,\|x\|)$ of center 0 and radius $\|x\|$, such that for every $g$ in $\mathcal{E}\left(\mathbb{R}^{d}\right)$, we have

$$
V_{k}(g)(x)=\int_{\mathbb{R}^{d}} g(y) d \mu_{x}(y) .
$$

Proof. From (3.1), (4.21) we have

$$
V_{k}(g)(x)=\left\langle K_{x}, g\right\rangle \geq 0,
$$

with $K_{x}$ in $\mathcal{E}^{\prime}\left(\mathbb{R}^{d}\right)$ such that $\operatorname{supp} K_{x} \subset B(0,\|x\|)$.

Thus from (4.24) and Theorem $\mathrm{V}$ of [8], p. 29, the distribution $K_{x}$ is a positive measure on $\mathfrak{m}$ denoted by $\mu_{x}$ with support in $B(0,\|x\|)$.

Corollary 4.9. Let $\mathfrak{m}$ be the $\sigma$-algebra in $\mathbb{R}^{d}$ which contains all Borel sets in $\mathbb{R}^{d}$. Then we have the following:

i) For each $y \in \mathbb{R}^{d}$ there exists a unique positive measure $\nu_{y}^{W}$ on $\mathfrak{m}$ such that for ever $f$ in $\mathcal{D}\left(\mathbb{R}^{d}\right)^{W}$, we have

$$
{ }^{t} V_{k}^{W}(f)(y)=\int_{\mathbb{R}^{d}} f(x) d \nu_{y}^{W}(x),
$$

with

$$
\nu_{y}^{W}=\frac{1}{|W|} \sum_{w \in W} \nu_{w y}
$$

where $\nu_{y}$ is the measure given by (4.19). 
ii) For each $x \in \mathbb{R}^{d}$ there exists a unique positive measure $\mu_{x}^{W}$ on $\mathfrak{m}$ with support in the ball $B(0,\|x\|)$, such that for every $g$ in $\mathcal{E}\left(\mathbb{R}^{d}\right)^{W}$, we have

$$
V_{k}^{W}(g)(x)=\int_{\mathbb{R}^{d}} g(y) d \mu_{x}^{W}(y),
$$

with

$$
\mu_{x}^{W}=\frac{1}{|W|} \sum_{w \in W} \mu_{w x}
$$

where $\mu_{x}$ is the measure given by (4.23).

Corollary 4.10. We have the following:

i) The Opdam-Cherednik kernel $G_{\lambda}(x), x \in \mathbb{R}^{d}, \lambda \in \mathbb{C}^{d}$, and the HeckmanOpdam kernel $F_{\lambda}(x), x \in \mathbb{R}^{d}, \lambda \in \mathbb{C}^{d}$, possess the Laplace type integral representations

$$
\begin{aligned}
& G_{\lambda}(x)=\int_{\mathbb{R}^{d}} e^{-i\langle\lambda, y\rangle} d \mu_{x}(y) . \\
& F_{\lambda}(x)=\int_{\mathbb{R}^{d}} e^{-i\langle\lambda, y\rangle} d \mu_{x}^{W}(y) .
\end{aligned}
$$

ii) We have

$$
\begin{aligned}
& \forall x \in \mathbb{R}^{d}, \forall \lambda \in \mathbb{R}^{d}, G_{i \lambda}(x)>0 . \\
& \forall x \in \mathbb{R}^{d}, \forall \lambda \in \mathbb{R}^{d}, F_{i \lambda}(x)>0 .
\end{aligned}
$$

iii) For all $x \in \mathbb{R}^{d}$, the functions $\lambda \rightarrow G_{\lambda}(x)$ and $\lambda \rightarrow F_{\lambda}(x)$ are positive definite.

\section{Proof.}

i) We deduce (4.29) from (4.23), (2.21), and we obtain (4.30) from (4.27), $(2.23)$.

ii) We get (4.31), (4.32) from (4.29), (4.30) and the positivity of the measures $\mu_{x}$ and $\mu_{x}^{W}$.

The relations (4.31), (4.32) have been proved in [6] by using another method.

iii) The relations (4.29), (4.30) imply the result. 


\section{Absolute Continuity of the Measures $\mu_{x}$ and $\nu_{y}$}

Notations. We denote by

- $B(c, a)$ the open ball of $\mathbb{R}^{d}$ of center $c \in \mathbb{R}^{d}$ and radius $a>0$, and by $\bar{B}(c, a)$ its closure.

- $\lambda$ the Lebesgue measure on $\mathbb{R}^{d}$.

Proposition 5.1. Let $y_{0} \in \mathbb{R}^{d}$ and $a>0$. We consider the sequence $\left\{g_{n}\right\}_{n \in \mathbb{N} \backslash\{0\}}$ of functions in $\mathcal{D}\left(\mathbb{R}^{d}\right)$, positive, increasing such that:

$$
\forall n \in \mathbb{N} \backslash\{0\}, \operatorname{suppg}_{n} \subset \bar{B}\left(y_{0}, a\right), \forall y \in B\left(y_{0}, a-\frac{1}{n}\right), g_{n}(y)=1,
$$

and

$$
\forall y \in \mathbb{R}^{d}, \lim _{n \rightarrow+\infty} g_{n}(y)=1_{B\left(y_{0}, a\right)}(y),
$$

where $1_{B\left(y_{0}, a\right)}$ is the characteristic function of the ball $B\left(y_{0}, a\right)$. We have

$$
\begin{aligned}
\forall x \in \mathbb{R}^{d}, \lim _{n \rightarrow+\infty} V_{k}\left(g_{n}\right)(x) & =\lim _{n \rightarrow+\infty} \int_{\mathbb{R}^{2}} g_{n}(y) d \mu_{x}(y) \\
& =\int_{\mathbb{R}^{d}} 1_{B\left(y_{0}, a\right)}(y) d \mu_{x}(y) .
\end{aligned}
$$

The function $x \rightarrow \mu_{x}\left(B\left(y_{0}, a\right)\right)=\int_{\mathbb{R}^{d}} 1_{B\left(y_{0}, a\right)}(y) d \mu_{x}(y)$, which can also be denoted by $V_{k}\left(1_{B\left(y_{0}, a\right)}\right)(x)$ is defined almost every where on $\mathbb{R}^{d}$ (see [1], p. 17), measurable and for all $f$ in $\mathcal{D}\left(\mathbb{R}^{d}\right)$, we have

$$
\int_{\mathbb{R}^{d}} \mu_{x}\left(B\left(y_{0}, a\right)\right) f(x) \mathcal{A}_{k}(x) d x=\int_{B\left(y_{0}, a\right)}{ }^{t} V_{k}(f)(y) d y .
$$

Proof. For all $n \in \mathbb{N} \backslash\{0\}$, the function $V_{k}\left(g_{n}\right)$ belongs to $\mathcal{E}\left(\mathbb{R}^{d}\right)$. Then we obtain the results of this proposition from the continuity of the operator $V_{k}$ from $\mathcal{E}\left(\mathbb{R}^{d}\right)$ into itself, the monotonic convergence theorem and the relation (3.4).

Remark 5.2. There exists $a \sigma$-algebra $\mathfrak{m}$ in $\mathbb{R}^{d}$ which contains all Borel sets in $\mathbb{R}^{d}$. Then for all $E \in \mathfrak{m}$, the function $x \rightarrow \mu_{x}(E)$ is defined almost every where on $\mathbb{R}^{d}$, measurable and we have the following relation

$$
\int_{\mathbb{R}^{d}} \mu_{x}(E) f(x) \mathcal{A}_{k}(x) d x=\int_{E}{ }^{t} V_{k}(f)(y) d y, \quad f \in \mathcal{D}\left(\mathbb{R}^{d}\right) .
$$


Proposition 5.3. Let $x_{0} \in \mathbb{R}^{d}$ and $a>0$. We consider the sequence $\left\{f_{n}\right\}_{n \in \mathbb{N} \backslash\{0\}}$ of functions in $\mathcal{D}\left(\mathbb{R}^{d}\right)$, positive, increasing such that:

$$
\forall n \in \mathbb{N} \backslash\{0\}, \operatorname{supp}_{n} \subset \bar{B}\left(x_{0}, a\right), \forall x \in B\left(x_{0}, a-\frac{1}{n}\right), f_{n}(x)=1,
$$

and

$$
\forall x \in \mathbb{R}^{d}, \lim _{n \rightarrow+\infty} f_{n}(x)=1_{B\left(x_{0}, a\right)}(x),
$$

where $1_{B\left(x_{0}, a\right)}$ is the characteristic function of the ball $B\left(x_{0}, a\right)$. We have

$$
\begin{aligned}
\forall y \in \mathbb{R}^{d}, \lim _{n \rightarrow+\infty}{ }^{t} V_{k}\left(f_{n}\right)(y) & =\lim _{n \rightarrow+\infty} \int_{\mathbb{R}^{d}} f_{n}(x) d \nu_{y}(x) \\
& =\int_{\mathbb{R}^{d}} 1_{B\left(x_{0}, a\right)}(x) d \nu_{y}(x) .
\end{aligned}
$$

The function $y \rightarrow \nu_{y}\left(B\left(x_{0}, a\right)\right)=\int_{\mathbb{R}^{d}} 1_{B\left(x_{0}, a\right)}(x) d \nu_{y}(x)$, which can also be denoted by ${ }^{t} V_{k}\left(1_{B\left(x_{0}, a\right)}\right)(y)$ is defined almost every where on $\mathbb{R}^{d}$ (see [1], p. 17), measurable and for all $g$ in $\mathcal{E}\left(\mathbb{R}^{d}\right)$, we have

$$
\int_{\mathbb{R}^{d}} \nu_{y}\left(B\left(x_{0}, a\right)\right) g(y) d y=\int_{B\left(x_{0}, a\right)} V_{k}(g)(x) \mathcal{A}_{k}(x) d x .
$$

Proof. For all $n \in \mathbb{N} \backslash\{0\}$, the function ${ }^{t} V_{k}\left(f_{n}\right)$ belongs to $\mathcal{D}\left(\mathbb{R}^{d}\right)$. Then the continuity of the operator ${ }^{t} V_{k}$ from $\mathcal{D}\left(\mathbb{R}^{d}\right)$ into itself, the monotonic convergence theorem and the relation (3.4) imply the results of this proposition.

\subsection{Absolute Continuity of the Measure $\nu_{y}$}

The purpose of this subsection is to prove that for all $y \in \mathbb{R}^{d}$, the measure $\nu_{y}$ is absolute continuous will respect to the Lebesgue measure on $\mathbb{R}^{d}$.

Proposition 5.4. For $x \in \mathbb{R}_{r e g}^{d}$, there exists a unique positive function $\mathcal{K}(x,$.$) integrable with respect to the Lebesgue measure \lambda$, and a positive measure $\mu_{x}^{s}$ on $\mathbb{R}^{d}$ such that for every Borel set $E$, we have

$$
\mu_{x}(E)=\int_{E} \mathcal{K}(x, y) d y+\mu_{x}^{s}(E) .
$$

Proof. We deduce (5.4) from (1.1) and Theorem 6.9 of [6], p.129-130, and Theorem 8.6 and its Corollary of [6]. p. 166.

\section{Remarks 5.5.}


i) The supports of the function $y \rightarrow \mathcal{K}(x, y)$ and the measure $\mu_{x}^{s}$ are contained in the ball $B(0,\|x\|)$.

ii) The measures $\mu_{x}^{s}$ and the Lebesgue measure $\lambda$ are mutually singular.

iii) From Theorem 8.6, p. 166 and Definition 8.3, p.164, of [6], we have

$$
\mathcal{K}(x, y)=\lim _{a \rightarrow 0} \frac{\mu_{x}(B(y, a))}{\lambda(B(y, a))} .
$$

Proposition 5.6. We consider $x \in \mathbb{R}_{\text {reg }}^{d}$ and a positive function $f$ in $\mathcal{D}\left(\mathbb{R}^{d}\right)$ with support contained in the ball $\bar{B}(0, R), R>0$.

i) For all Borel set E, we have

$$
\int_{E} \mathcal{N}^{f}(y) d y=\int_{\bar{B}(0, R)} \mu_{x}^{s}(E) f(x) \mathcal{A}_{k}(x) d x,
$$

where

$$
\mathcal{N}^{f}(y)={ }^{t} V_{k}(f)(y)-\int_{\bar{B}(0, R)} \mathcal{K}(x, y) f(x) \mathcal{A}_{k}(x) d x
$$

ii) We have

$$
\forall y \in \mathbb{R}^{d}, \mathcal{N}^{f}(y) \geq 0
$$

\section{Proof.}

i) By using the relations (5.2), (5.5), we obtain

$$
\begin{aligned}
\int_{E}{ }^{t} V_{k}(f)(y) d y & =\int_{\bar{B}(0, R)} \mu_{x}(E) f(x) \mathcal{A}_{k}(x) d x \\
& =\int_{\bar{B}(0, R)}\left[\int_{E} \mathcal{K}(x, y) d y+\mu_{x}^{s}(E)\right] f(x) \mathcal{A}_{k}(x) d x
\end{aligned}
$$

We deduce (5.6) by applying Fubini-Tonelli's theorem to the second member.

ii) From the relation (5.6), the positivity of the measure $\mu_{x}^{s}$ implies that for all Borel set $E$, we have

$$
\int_{E} \mathcal{N}^{f}(y) d y \geq 0
$$


Thus

$$
\forall y \in \mathbb{R}^{d}, \mathcal{N}^{f}(y) \geq 0
$$

Proposition 5.7. The measure $\Lambda^{f}$ on $\mathbb{R}^{d}$, given for all Borel set $E$ by

$$
\Lambda^{f}(E)=\int_{E} \mathcal{N}^{f}(y) d y,
$$

is positive and bounded.

Proof.

- The relation (5.8) gives the positivity of the measure $\Lambda^{f}$.

- From the relations (5.9), (5.6), for all Borel set $E$ we have

$$
\Lambda^{f}(E) \leq \int_{\bar{B}(0, R)}\left\|\mu_{x}^{s}\right\| f(x) \mathcal{A}_{k}(x) d x .
$$

On the other hand by using (5.4), we obtain for $x \in \mathbb{R}_{\text {reg }}^{d}$,

$$
\mu_{x}^{s}(E) \leq \mu_{x}(E)
$$

thus

$$
\left\|\mu_{x}^{s}\right\| \leq\left\|\mu_{x}\right\|=1
$$

By using this result, the relation (5.10) implies that for all Borel set $E$, we have

$$
\Lambda^{f}(E) \leq M_{f}
$$

where

$$
M_{f}=\int_{\bar{B}(0, R)} f(x) \mathcal{A}_{k}(x) d x .
$$

Then the measure $\Lambda^{f}$ is bounded.

Proposition 5.8. Let $x \in \mathbb{R}_{\text {reg }}^{d}$ and $f$ be the function given in Proposition 5.6.

i) For all Borel set $E$ we have

$$
\Lambda^{f}(E)=0
$$

ii) For $y \in \mathbb{R}^{d}$, we have

$$
{ }^{t} V_{k}(f)(y)=\int_{\bar{B}(0, R)} \mathcal{K}(x, y) f(x) \mathcal{A}_{k}(x) d x .
$$




\section{Proof.}

i) From the relations (5.9), (5.6), for all Borel set $E$ the measure $\Lambda^{f}$ possesses also the following form

$$
\Lambda^{f}(E)=\int_{\bar{B}(0, R)} \mu_{x}^{s}(E) f(x) \mathcal{A}_{k}(x) d x .
$$

On the other hand from Proposition 5.7 the measure $\Lambda^{f}$ is absolute continuous with respect to the Lebesgue measure $\lambda$ and from Remark 5.5 ii) the measure $\mu_{x}^{s}, x \in \bar{B}(0, R)$, and the Lebesgue measure $\lambda$ are mutually singular. Then from Proposition 6.8 (f), p.129, of [6], the measure $\Lambda^{f}$ and $\mu_{x}^{s}, x \in \bar{B}(0, R)$, are mutually singular. By using the definition of measures mutually singular (see p. 128 of [6]), we deduce (5.11) from (5.13).

ii) By using the i) and (5.9), (5.7), we obtain (5.12).

Theorem 5.9. For all $f$ in $\mathcal{D}\left(\mathbb{R}^{d}\right)$ we have

$$
\forall y \in \mathbb{R}^{d},{ }^{t} V_{k}(f)(y)=\int_{\mathbb{R}^{d}} \mathcal{K}(x, y) f(x) \mathcal{A}_{k}(x) d x .
$$

Proof. We obtain (5.14) by writing $f=f^{+}-f^{-}$and by using Proposition 5.8 ii).

Remark 5.10. Theorem 5.9 shows that for all $y \in \mathbb{R}^{d}$ the measure $\nu_{y}$ given by the relation (1.2), is absolute continuous with respect to the measure $\mathcal{A}_{k}(x) d x$. More precisely we have

$$
d \nu_{y}(x)=\mathcal{K}(x, y) \mathcal{A}_{k}(x) d x .
$$

\subsection{Absolute Continuity of the Measure $\mu_{x}$}

The purpose of this subsection is to prove that for all $x \in \mathbb{R}_{r e g}^{d}$ the measure $\mu_{x}$ is absolute continuous with respect to the Lebesgue measure on $\mathbb{R}^{d}$.

Theorem 5.11. For all $g$ in $\mathcal{E}\left(\mathbb{R}^{d}\right)$ and $x_{0} \in \mathbb{R}_{\text {reg }}^{d}$, we have

$$
V_{k}(g)\left(x_{0}\right)=\int_{\mathbb{R}^{d}} \mathcal{K}\left(x_{0}, y\right) g(y) d y .
$$


Proof. By writing $g=g^{+}-g^{-}$, it suffices to prove the theorem for $g$ positive.

From the relation (5.3) we have

$$
\frac{1}{\lambda\left(B\left(x_{0}, a\right)\right)} \int_{B\left(x_{0}, a\right)} V_{k}(g)(x) \mathcal{A}_{k}(x) d x=\int_{\mathbb{R}^{d}} g(y) \frac{\nu_{y}\left(B\left(x_{0}, a\right)\right)}{\lambda\left(B\left(x_{0}, a\right)\right)} d y .
$$

By using the relation (5.14), and by applying Fubini-Tonelli's theorem to the second member of (5.17), we obtain

$$
\begin{aligned}
\frac{1}{\lambda\left(B\left(x_{0}, a\right)\right)} \int_{B\left(x_{0}, a\right)} V_{k}(g)(x) \mathcal{A}_{k}(x) d x= \\
\quad \frac{1}{\lambda\left(B\left(x_{0}, a\right)\right)} \int_{B\left(x_{0}, a\right)}\left[\int_{\mathbb{R}^{d}} \mathcal{K}(x, y) g(y) d y\right] \mathcal{A}_{k}(x) d x .
\end{aligned}
$$

By applying the relation (2) of [6], p.168, to the two members of this relation we get

$$
\mathcal{A}_{k}\left(x_{0}\right) V_{k}(g)\left(x_{0}\right)=\mathcal{A}_{k}\left(x_{0}\right) \int_{\mathbb{R}^{d}} \mathcal{K}\left(x_{0}, y\right) g(y) d y,
$$

as

$$
\mathcal{A}_{k}\left(x_{0}\right) \neq 0 \Leftrightarrow x_{0} \in \mathbb{R}_{r e g}^{d},
$$

thus for $x_{0} \in \mathbb{R}_{r e g}^{d}$, we have

$$
V_{k}(g)\left(x_{0}\right)=\int_{\mathbb{R}^{d}} \mathcal{K}\left(x_{0}, y\right) g(y) d y .
$$

Remark 5.12. From Theorem 5.11 and the relation (1.1) we deduce that for all $x \in \mathbb{R}_{r e g}^{d}$ the measure $\mu_{x}$ is absolute continuous with respect to the Lebesgue measure on $\mathbb{R}^{2}$. More precisely, we have

$$
d \mu_{x}(y)=\mathcal{K}(x, y) d y .
$$

\section{Corollary 5.13.}

i) For all $\lambda \in \mathbb{C}^{2}$ and $x \in \mathbb{R}_{\text {reg }}^{d}$, we have

$$
G_{\lambda}(x)=\int_{\mathbb{R}^{d}} \mathcal{K}(x, y) e^{-i\langle\lambda, y\rangle} d y .
$$

ii) For all $x \in \mathbb{R}_{\text {reg }}^{d}$, we have

$$
\int_{\mathbb{R}^{d}} \mathcal{K}(x, y) d y=1 .
$$


iii) For all $x \in \mathbb{R}_{\text {reg }}^{d}$, we have

$$
\operatorname{supp\mathcal {K}}(x, .) \subset \bar{B}(0,\|x\|) .
$$

Proof. We deduce the results of this Corollary from (1.1), (5.19), and Theorem 5.11.

Theorem 5.14. We have

$$
\forall x \in \mathbb{R}_{r e g}^{d}, \lim _{\|\lambda\| \rightarrow+\infty} G_{\lambda}(x)=0 .
$$

Proof. From the relation (5.20) the function $\mathcal{K}(x,$.$) is integrable on \mathbb{R}^{d}$ with respect to the Lebesgue measure on $\mathbb{R}^{d}$. Then we deduce (5.22) from the relation (5.19) and Riemann-Lebesgue Lemma for the usual Fourier transform on $\mathbb{R}^{d}$.

\subsection{Absolute Continuity of the Measures $\nu_{y}^{W}$ and $\mu_{x}^{W}$}

Theorem 5.15. For all $f$ in $\mathcal{D}\left(\mathbb{R}^{d}\right)^{W}$, we have

$$
\forall y \in \mathbb{R}^{d},{ }^{t} V_{k}^{W}(f)(y)=\int_{\mathbb{R}^{d}} \mathcal{K}^{W}(x, y) f(x) \mathcal{A}_{k}(x) d x,
$$

where $\mathcal{K}^{W}(x, y)$ is the function given by

$$
\mathcal{K}^{W}(x, y)=\frac{1}{|W|^{2}} \sum_{w, w^{\prime} \in W} \mathcal{K}\left(w x, w^{\prime} y\right)
$$

Proof. The relations (1.4), (1.6) and Theorem 5.9 imply the relations (5.23), (5.30).

Theorem 5.16. For all $g$ in $\mathcal{E}\left(\mathbb{R}^{d}\right)^{W}$, we have

$$
\forall x \in \mathbb{R}_{r e g}^{d}, V_{k}^{W}(g)(x)=\int_{\mathbb{R}^{d}} \mathcal{K}^{W}(x, y) g(y) d y,
$$

where $\mathcal{K}^{W}(x, y)$ is the function given by the relation (5.24).

Proof. We deduce (5.25) from the relations (1.3), (1.5) and Theorem 5.11.

Remark 5.17. Theorems 5.15, 5.16 show that the measures $\nu_{y}^{W}, y \in \mathbb{R}^{d}$ and $\mu_{x}^{W}, x \in \mathbb{R}_{r e g}^{d}$, given respectively by the relations (1.4), (1.3), are absolute 
continuous with respect to the Lebesgue measure on $\mathbb{R}^{d}$. More precisely we have

$$
d \nu_{y}^{W}(x)=\mathcal{K}^{W}(x, y) \mathcal{A}_{k}(x) d x
$$

and

$$
d \mu_{x}^{W}(y)=\mathcal{K}^{W}(x, y) d y
$$

Corollary 5.18.

i) For all $\lambda \in \mathbb{C}^{d}$ and $x \in \mathbb{R}_{\text {reg }}^{d}$, we have

$$
F_{\lambda}(x)=\int_{\mathbb{R}^{d}} \mathcal{K}^{W}(x, y) e^{-i\langle\lambda, y\rangle} d y
$$

ii) For all $x \in \mathbb{R}_{\text {reg }}^{d}$, we have

$$
\int_{\mathbb{R}^{d}} \mathcal{K}^{W}(x, y) d y=1
$$

iii) For all $x \in \mathbb{R}_{\text {reg }}^{d}$, we have

$$
\operatorname{supp}^{W}(x, .) \subset \bar{B}(0,\|x\|)
$$

Proof. The relations (1.3), (4.30) and Theorem 5.16, imply the results of this Corollary.

Theorem 5.19. We have

$$
\forall x \in \mathbb{R}_{r e g}^{d}, \lim _{\|\lambda\| \rightarrow+\infty} F_{\lambda}(x)=0 .
$$

Proof. We deduce (5.31) from the relations (5.28), (5.29) and RiemannLebesgue Lemma for the usual Fourier transform on $\mathbb{R}^{d}$.

\section{References}

[1] N. Bourbaki, Elements de Mathématiques, Fasicule XXI. Integration des mesures. Chapitre 5, Hermann, Paris, 1967.

[2] I. Cherednik, A unification of Knizhnik-Zamslodchnikov equations and Dunkl operators via affine Hecke algebras, Invent. Math., 106 (1990), 411432. 
[3] L. Gallardo and K. Trimèche, Positivity of the Jacobi-Cherednik intertwining operator and its dual, Adv. Pure Appl. Math., 1 (2010), 163-194.

[4] G.J. Heckman and E.M. Opdam, Root systems and hypergeometric functions: I, Compos. Math., 64 (1987), 329-352.

[5] E.M. Opdam, Harmonic analysis for certain representations of graded Hecke algebras, Acta Math., 175 (1995), 75-121.

[6] W. Rudin, Real and Complex Analysis, Second Edition, McGraw-Hill, 1974.

[7] B. Schapira, Contribution to the hypergeometric function theory of Heckman and Opdam: Sharp estimates, Schwartz spaces, heat kernel, Geom. Funct. Anal., 18 (2008), 222-250.

[8] L. Schwartz, Theorie des distributions, Hermann, Paris, 1966.

[9] K. Trimèche, The trigonometric Dunkl intertwining operator and its dual associated with the Cherednik operators and the Heckman-Opdam theory, Adv. Pure Appl. Math., 1 (2010), 293-323.

[10] K. Trimèche, Harmonic analysis associated with the Cherednik operators and the Heckman-Opdam theory, Adv. Pure Appl. Math., 2 (2011), 223-46.

[11] K. Trimèche, Positivity of the transmutation operators associated with a Cherednik type operator on the real line, Adv. Pure Appl. Math., 3 (2012), 361-376.

[12] K. Trimèche, The harmonic analysis associated to the Heckman-Opdam theory and its application to a some root system of type $B C_{d}$, Preprint Faculty of Sciences of Tunis, 2015. 
